\title{
La fonction du savoir imaginaire dans La Vie mode d'emploi de Georges Perec
}

Shuichiro Shiotsuka

\section{Q OpenEdition \\ 1 Journals}

Édition électronique

URL : http://journals.openedition.org/aes/312

DOI : 10.4000/aes.312

ISSN : 2258-093X

Éditeur

Laboratoire LISAA

Référence électronique

Shuichiro Shiotsuka, "La fonction du savoir imaginaire dans La Vie mode d'emploi de Georges Perec », Arts et Savoirs [En ligne], 5 | 2015, mis en ligne le 09 novembre 2015, consulté le 01 mai 2019. URL

http://journals.openedition.org/aes/312; DOI : 10.4000/aes.312

Ce document a été généré automatiquement le 1 mai 2019.

Centre de recherche LISAA (Littératures SAvoirs et Arts) 


\title{
La fonction du savoir imaginaire dans La Vie mode d'emploi de Georges Perec
}

\author{
Shuichiro Shiotsuka
}

1 Dans son livre L'Érudition imaginaire, Nathalie Piégay-Gros fait souvent mention de La Vie mode d'emploi de Georges Perec, car, du simple titre de l'ouvrage aux propositions les plus hétérodoxes, le roman multiplie les savoirs imaginaires. Si la plupart de ces savoirs existent sous forme de détails fragmentaires, ne formant pas de système cohérent, ils n'en jouent pas moins dans plusieurs chapitres un rôle important en tant que moteurs du récit. Comme le remarque très pertinemment Nathalie Piégay-Gros, "pour Perec, le savoir semble n'avoir pour fin que le romanesque. Il jette de l'huile sur le foyer de l'imagination pour mieux l'enflammer; curieux usage de l'érudition, mis au service du romanesque, de ses passions juvéniles, aventureuses et sentimentales! » ${ }^{1}$ Le romancier s'est expliqué sur son usage de la pseudo-érudition en ces termes :

le texte n'est pas producteur de savoir, mais producteur de fiction, de fiction de savoir, de savoir-fiction. Quand je dis que je voudrais que mes textes soient informés par les savoirs contemporains comme les romans de Jules Verne le furent par la science de son époque, cela veut dire que je voudrais qu'ils interviennent dans l'élaboration de mes fictions, non pas en tant que vérité, mais en tant que matériel, ou machinerie, de l'imaginaire. ${ }^{2}$

2 Attardons-nous un moment sur la signification du mot «machinerie » dans ces propos rapportés d'ailleurs par Nathalie Piégay-Gros à l'appui de sa thèse. Se pourrait-il que, dans le roman perecquien, les savoirs ne servent pas seulement à stimuler l'imagination ou à nourrir l'anecdote, mais fonctionnent comme rouages de la machinerie ? Compte tenu de la complexité des systèmes de contraintes qui sous-tendent La Vie mode d'emploi, on est tenté de croire que ce roman constitue une véritable machinerie. Alors, quel est le rôle de ces savoirs dans la stratégie globale de l'écriture du livre ? L'objectif de cet article est de répondre à cette question en considérant plusieurs chapitres dont la diégèse met en scène un savoir particulier. 
3 Le chapitre II du roman, qui raconte l'épisode de l'archéologue Fernand de Beaumont, nous servira de point de départ: Beaumont a entrepris, sur le site de la cité légendaire Lebtit, des fouilles archéologiques qui ont échoué, le conduisant au suicide. Christelle Reggiani suggère que cette anecdote peut se lire comme un avertissement au lecteur sur les limites d'une fouille archéologique visant à "déterrer " les contraintes qui soustendent le texte ${ }^{3}$. En outre, à l'intérieur de l'épisode, l'archéologue localise l'emplacement de la citadelle, en interprétant de manière métaphorique la légende sur Lebtit ${ }^{4}$ démarche qui tend à nous inviter à une lecture allégorique du récit de fouille.

4 Si l'archéologie est une exploration des temps lointains, l'ethnographie s'intéresse aux habitants de contrées lointaines. En fait, l'ethnographie, qui fournit la matière du chapitre XXV, fait pendant à l'archéologie : l'ethnographe Marcel Appenzzell est parti en expédition à Sumatra dans le cadre d'un travail de recherche sur un peuple fantôme, les Orang-Kubus ; mais, devant l'impossibilité d'établir le moindre contact significatif avec eux, l'entreprise a échoué. Appenzzell a néanmoins observé que les Kubus parlaient une langue dont le vocabulaire était extrêmement réduit et ne dépassait pas quelques dizaines de mots :

il se demanda si, à l'instar de leurs lointains voisins les Papouas, les Kubus n'appauvrissaient pas volontairement leur vocabulaire, supprimant des mots chaque fois qu'il y avait un mort dans le village. Une des conséquences de ce fait était qu'un même mot désignait un nombre de plus en plus grand d'objets. ${ }^{5}$

Cette caractéristique de leur langue est comparable à celle de l'écriture perecquienne, en ce sens que certaines expressions apparemment banales dans La Vie mode d'emploi peuvent désigner plus d'une chose à la fois. Ainsi quand il réapparut après presque six ans d'expédition, Appenzzell était vêtu d'« une espèce de pantalon fait d'innombrables petits bouts de tissus cousus ensemble $»^{6}$, détail significatif au niveau de la diégèse, mais qui pourrait tout aussi bien désigner la manière dont se construit le roman, qui est justement un assemblage de morceaux. La langue des Kubus a paru à l'ethnologue d'autant plus déconcertante qu'elle cache l'altérité absolue sous une apparence de banalité, choc psychologique qui expliquerait, nous semble-t-il, qu'à son retour en Europe, il ait " pratiquement perdu l'usage de la parole $»^{7}$.

6 Du point de vue qui nous occupe, si le suicide de Beaumont nous avertit des limites d'une « lecture archéologique », l'échec d'Appenzzell nous retient peut-être de tomber dans une lecture de type ethnographique. En quoi celle-ci consisterait-elle ? L'ethnographie est une activité qui a pour but de comprendre "l'autre ", en tant que distinct de l'observateur. Si l'observateur s'identifie à lui, partageant longtemps la vie d'une tribu, il ne pourra pas rendre compte objectivement de son expérience parmi les indigènes. Mais s'il garde une certaine distance, les indigènes lui demeurent tant étrangers qu'inaccessibles. La «lecture ethnographique » consisterait donc à prétendre comprendre un texte parfois fermé au lecteur. L'épisode de la tribu qui fuit l'ethnographe nous inciter à nous méfier d'une lecture au premier degré et de l'interprétation univoque d'un récit.

7 Si la mise en scène de certaines sciences humaines comme l'archéologie et l'ethnographie peut s'interpréter comme avertissement au lecteur, qu'en est-il des sciences exactes? Dans le chapitre LXII, le chimiste Wehsal tente de reproduire des techniques, mises au point sous le III ${ }^{e}$ Reich, qui consistent à fabriquer de l'essence à partir du lignite ou de la tourbe. À la différence de Beaumont et d'Appenzzell, le chimiste réussit son entreprise mais il est acculé au suicide, "accusé d'avoir tenté de livrer des secrets "d'importance stratégique" à une puissance étrangère $»^{8}$. Malgré l'importance de ses découvertes, celles- 
ci sont, au début, sous-estimées par les experts : selon eux, il s'agirait de techniques "lourdes, inélégantes et dépassées ", nécessitant des "dispositifs [trop] encombrants". Un personnage va jusqu'à considérer la démarche de Wehsal comme l'« exemple typique de la bêtise scientifique ». Ainsi, ce qui est en cause dans ce chapitre, ce n'est pas l'issue des recherches, mais l'accueil qui leur est fait.

Comment ne pas voir dans les techniques mises au point par le chimiste quelques équivalences avec les dispositifs générateurs de La Vie mode d'emploi ? Les remarques des experts telles que «techniques lourdes » ou "dispositifs encombrants » ne pourraientelles pas en effet s'appliquer au programme qui a servi à générer le roman et évoquer une certaine critique à l'encontre des constructions oulipiennes? Par ailleurs, le principe même de la pétrochimie - combinaison, synthèse ${ }^{9}$ - peut se lire comme désignation métaphorique du recours à des algorithmes imbriqués les uns dans les autres pour établir une liste d'éléments à inclure dans chaque chapitre et à combiner pour générer du récit.

9 De tous les savoirs, c'est toutefois l'activité lexicographique qui se rapproche le plus de l'écriture. Dans le chapitre LX, le personnage de Chinoc projette de « rédiger un grand dictionnaire des mots oubliés $»^{10}$. L'incertitude quant à la prononciation exacte du nom Chinoc, aussi bien que son origine polonaise, nous rappelle déjà les propos de Perec sur son propre patronyme ${ }^{11}$. En quel sens peut-on dire que le "grand dictionnaire des mots oubliés ", conçu par le double de Perec, évoque La Vie mode d'emploi? Le trait commun entre les deux livres, c'est la fonction de conservation. Michael Sheringham fait remarquer un caractère de " capsule temporelle » du roman perecquien rassemblant tous les objets quotidiens et ordinaires de la dernière moitié du $\mathrm{XX}^{\mathrm{e}}$ siècle ${ }^{12}$. La Vie mode d'emploi est donc une sorte de musée des objets oubliés, mais en plus, ce roman est réellement un « grand dictionnaire des mots oubliés ${ }^{13}$, puisqu'il contient bon nombre de mots rares, de mots archaïques, de termes techniques. L'entreprise du lexicographe met en relief cet acte conservatoire du roman.

10 Comme la lexicographie, l'histoire est une discipline qui s'intéresse aux documents d'archives. Le chapitre XXII raconte une histoire d'escroquerie postulant l'existence réelle du Saint Vase, où les uns trompent les autres, où le vrai devient le faux, et inversement. Il est évident qu'une pareille manœuvre calque celle de la lecture ou de la création problématique qui déborde toutefois le cadre de notre propos. On en restera donc à des considérations historiques : au cours de la recherche du Saint Vase effectuée à la fin du XIX ${ }^{e}$ siècle par un professeur d'histoire ancienne sont découverts des documents datant du XVIII ${ }^{e}$ siècle, dans lesquels Jean-Baptiste Rousseau recense des reliques conservées jusqu'à cette époque. S'ensuit toute une série d'enchâssements. Comme l'indique Isabelle Dangy-Scaillierez, le livre ancien nommé "Quarli», livre-kangourou pourvu d'une poche ventrale, fonctionne comme la matrice du récit ${ }^{14}$, et dans la foulée, comme celle du roman qui englobe des chambres contenant chacune des anecdotes multiformes. Par ailleurs, comme il se doit pour l'histoire du Saint Vase, les recherches s'étendent de la Renaissance jusqu'à la fin du XIX $X^{e}$ siècle, la scène se passe en Savoie, aux Pays-Bas et à New York. La vaste étendue spatio-temporelle de cet épisode ressemble à celle de La Vie mode d'emploi, toutes proportions gardées.

$11 \mathrm{Au}$ fond, le roman et l'histoire ont cela en commun qu'ils font apparaitre, par le biais du langage, des personnes et des objets inexistants. Cela revient à dire que la démarche représentée dans ce chapitre a davantage d'affinités avec le romanesque que l'archéologie ou la pétrochimie. C'est pourquoi, nous semble-t-il, en mettant en scène des recherches historiques, Perec ne se contente pas de les lier à La Vie mode d'emploi, mais finit par les 
contester dans la dernière phase de l'épisode: en révélant que tous les documents attestant l'existence réelle du Saint Vase sont faux, il expose la fictionnalité même du récit.

De même que la recherche historique accuse le mensonge du roman, de même la cartographie fonctionne comme un dispositif visant à créer un effet de distanciation. Dans la première moitié du chapitre LXXX apparaissent trois cartes particulières décorant la chambre du personnage. La première est une vieille carte en mauvais état dont le déchiffrement fut à l'origine d'une controverse en matière d'onomastique. Comme le fait remarquer Andrée Chauvin, la reconstitution de l'inscription lacunaire est comparable à la solution d'un puzzle ou des mots croisés, ces motifs emblématiques de $L a$ Vie mode d'emploi ${ }^{15}$. Car, afin de choisir la solution correcte parmi plusieurs possibilités, il faut écarter les idées préconçues et faire basculer la perception, ainsi que Perec l'explique dans le préambule du roman. De fait, on sait bien que le basculement de la perception constitue l'un des thèmes principaux de son œuvre qui contient bon nombre de descriptions hyperréalistes à la manière du trompe-l'œil et des "détails métatexuels » dont la perception change selon l'optique adoptée. Or, cette vieille carte partiellement endommagée a ceci de particulier que «le nord n'est pas en haut [...] mais en bas »" détruisant également la perception habituelle: en ce sens, elle peut incarner l'un des principes de La Vie mode d'emploi.

13 La deuxième est une carte du Japon datant du XVII ${ }^{e}$ siècle, qui présente également des singularités d'orientation. Mais l'essentiel est que «les noms des soixante-six provinces impériales sont, pour la première fois, donnés en idéogrammes japonais et transcrits en caractères latins $»^{17}$. On pourrait considérer que la carte truffée de caractères est une figure emblématique de la création littéraire, parce que ce sont les lettres sur le papier blanc qui font sens tant dans l'écriture que dans la cartographie. Dans Espèces d'espaces, Perec illustre cette idée par l'exemple suivant :

L'espace commence ainsi, avec seulement des mots, des signes tracés sur la page blanche. Décrire l'espace : le nommer, le tracer, comme ces faiseurs de portulans qui saturaient les côtes de noms de ports, de noms de caps, de noms de criques, jusqu'à ce que la terre finisse par ne plus être séparée de la mer que par un ruban continu de texte. ${ }^{18}$

Dans ce cas, comme l'indique fort justement Jean-Luc Joly, l'activité démiurgique de l'écriture est assimilable à celle du cartographe ${ }^{19}$.

14 La troisième est une carte du Pacifique utilisée par les tribus de Papouasie: c'est un réseau de tiges de bambou qui indiquent les courants marins et les vents dominants, avec des coquillages représentant les îles et les écueils. Le narrateur commente ainsi ses particularités : «Par rapport aux normes adoptées aujourd'hui par tous les cartographes, cette "carte" semble une aberration. ${ }^{20}$ Mais, même si c'est le cas, les cartes "normales " sont-elles vraiment le miroir de la réalité ? En fait, le point de vue dominant toute la sphère d'une carte doit être omniprésent et irréel. On pourrait donc dire que la carte est une fiction. À vouloir être trop précis, l'on obtiendra cette carte borgésienne, « qui [a] le Format de l'Empire et qui coïncid[e] avec lui, point par point $»^{21}$. D'ailleurs, quelque aberrante qu'elle fût, la carte du Pacifique convenait tout à fait à la navigation maritime des tribus de Papouasie, de la même façon que le plan du métro londonien, qui «n'est absolument pas superposable à un plan de la ville de Londres $»^{22}$, est pratique.

Que dire alors de cette carte topologique? Il est possible qu'elle incarne la souplesse d'esprit, et en ce sens encourage le lecteur à une certaine flexibilité dans ses 
interprétations. Et il en est ainsi pour La Vie mode d'emploi, cette fiction qui représente un univers exagéré et déformé et qui, ce faisant, nous transmet quand même un grain de vérité sur l'essence de l'homme. Par ailleurs, en mettant en relief le côté négatif des «normes» rigides, la carte du Pacifique semble nous révéler l'un des secrets de fabrication du chapitre où elle figure. En effet, il s'est avéré que, dans la rédaction de ce chapitre, l'écrivain n'a presque pas respecté les contraintes préétablies. Comme le fait remarquer Andrée Chauvin, les cartes mêmes font leur apparition grâce au changement d'items dans la contrainte de «PEINTURE» : substitution de «cartes» au « mur nu » ${ }^{23}$. Dans le contexte de l'Oulipo, on appelle cette liberté dans le système le «clinamen ». Il sera donc possible de penser que non seulement la carte du Pacifique, mais aussi les deux autres cartes examinées ici sont des figures emblématiques du principe de clinamen. On l'a vu, les trois cartes du chapitre LXXX suggèrent respectivement certains aspects du mécanisme de La Vie mode d'emploi, y compris des propriétés du roman en général.

En conclusion, il nous semble que la proposition de Nathalie Piégay-Gros, qui nous a servi de point de départ, reste tout à fait pertinente. Pour Perec, en effet, « le savoir semble n'avoir pour fin que le romanesque», puisque le mot "romanesque» signifie non seulement «ce qui offre les caractères du roman traditionnel » mais également « ce qui est propre au roman ». De fait, les savoirs mis en fiction dans La Vie mode d'emploi archéologique, ethnographique, pétrochimique, lexicographique, historique et cartographique - peuvent s'interpréter comme autant de métaphores de la conception du roman et des techniques romanesques mises en œuvre par Perec.

Mais, pourquoi la science joue-t-elle ce rôle? Qu'est-ce qui lui vaut ce rôle ? C'est peutêtre la tendance catégorique de la science, parfois trop confiante en soi qui en est responsable. Dans son roman, Perec fait en sorte que toutes les recherches de la "vérité » se soldent par un échec. Par cela, il relativise peut-être non seulement la vérité scientifique mais également sa propre entreprise, qui a quelque chose de commun avec les recherches scientifiques : la volonté de totalité, le recours au système, etc. La science, en tant qu'activité systématisée et quelque peu catégorique, est donc une image de La Vie mode d'emploi, cette somme encyclopédique. En profitant du savoir scientifique pour se distancier de sa propre œuvre, Perec s'est sans doute montré lucide et froid, ce qui pourrait le distinguer d'autres avant-gardistes parfois trop dogmatiques. En ce sens, il représente le savoir à des fins tant romanesques qu'éthiques.

\section{NOTES}

1. Nathalie Piégay-Gros, L'Érudition imaginaire, Genève, Droz, 2009, p. 108.

2. Georges Perec, Jean-Marie Le Sidaner, «Entretien ", L'Arc, nº 76, 1979, p. 4.

3. Christelle Reggiani, Rhétoriques de la contrainte: Georges Perec - L'Oulipo, Éditions InterUniversitaires, 1999, p. 210.

4. Georges Perec, La Vie mode d'emploi, in Romans et récits, Paris, Le Livre de Poche, 2002, p. 664.

5. Ibid., p. 794

6. Ibid., p. 791. 
7. Perec, op. cit., p. 791.

8. Ibid., p. 1036.

9. «fabriquer de l'essence avec du lignite ou avec de la tourbe » (Ibid., p. 1036); « combiner un ion hydrogène et une molécule de monoxyde de carbone ( $\mathrm{CO}$ ) pour obtenir des molécules de pétrole » (Ibid., p. 1034).

10. Ibid., p. 1022.

11. Ibid., p. 1018-1019.

12. Michael Sheringham, Everyday Life: Theories and Practices from Surrealism to the Present, Londres, Oxford University Press, 2006 (réed. 2009), p. 283.

13. D'ailleurs, La Vie mode d'emploi enregistre de manière performative une partie du dictionnaire de Chinoc, dans laquelle figure un mot : «MITELLE (s. f.) [...] Chir. Écharpe pour soutenir le bras » (Ibid., p. 1024). Cette écharpe rappelle un souvenir important décrit dans $W$ ou le souvenir d'enfance et Je me souviens, si bien que ce roman constitue non seulement un dictionnaire à caractère public mais aussi un lexique personnel.

14. Isabelle Dangy-Scaillierez, L'Énigme criminelle dans les romans de Georges Perec, Paris, Honoré Champion, 2002, p. 116.

15. Andrée Chauvin, "Cartes et plans : représentation de l'espace et conditions de lecture ", Cahiers Georges Perec, $n^{\circ}$ 8, Castor Astral, 2004, p. 242.

16. Perec, op. cit., p. 1146.

17. Ibid.

18. Georges Perec, Espèces d'espaces, Paris, Galilée, 1974/2000 (nouvelle édition revue et corrigée), p. 26.

19. Jean-Luc Joly, "L'écriture cartographique de Georges Perec », Discursive geographies. Writing Space and Place in French, Amsterdam, Rodopi, 2005, p. 230.

20. Perec, La Vie mode d'emploi, op. cit., p. 1146.

21. Jorge Luis Borges, "De la rigueur de la science ", in Histoire universelle de l'infamie / Histoire de l'éternité, Union générale d'éditions, collection 10/18, 1994 (première édition française, 1951), p. 107.

22. Perec, La Vie mode d'emploi, op. cit., p. 1147.

23. Chauvin, op.cit., p. 246.

\section{RÉSUMÉS}

La Vie mode d'emploi de Georges Perec multiplie les savoirs imaginaires. Si la plupart de ces savoirs existent sous forme de détails fragmentaires, ne formant pas de système cohérent, ils n'en jouent pas moins dans plusieurs chapitres un rôle important en tant que moteurs du récit. Alors, quel est le rôle de ces savoirs dans la stratégie globale de l'écriture du livre ? L'objectif de cet article est de répondre à cette question en considérant plusieurs chapitres dont la diégèse met en scène un savoir particulier. Au terme de nos analyses, il s'avère que les savoirs mis en fiction dans $L a$ Vie mode d'emploi - archéologique, ethnographique, pétrochimique, lexicographique, historique et cartographique - peuvent s'interpréter comme autant de métaphores de la conception du roman et des techniques romanesques mises en œuvre par Perec. 
INDEX

Mots-clés : Perec (Georges), savoir, imaginaire, érudition

\section{AUTEUR}

SHUICHIRO SHIOTSUKA 